\title{
La comunidad al rescate: algunas reflexiones sobre las políticas estatales de activación comunitaria en Europa
}

\author{
Felix Arrieta
}

Departamento de Trabajo Social y Sociología, Universidad de Deusto felix.arrieta@deusto.es

\section{Ainhoa Izagirre}

Departamento de Trabajo Social y Sociología, Universidad de Deusto

\section{Mabel Segú}

Departamento de Trabajo Social y Sociología, Universidad de Deusto

\section{Martín Zuñiga}

Departamento de Trabajo Social y Sociología, Universidad de Deusto

\begin{abstract}
2008 urtetik aurrera gertatu izan diren transformazio sozialak (krisialdi ekonomikoa, familiaren egituretan eta lan-merkatuan sortutako aldaketak, etab.) gizartean agertutako arrisku sozial berriekin batera, Ongizate Estatuaren ahalmena zalantzan jarri dute, egoera honi aurre egiteko ziurgabetasuntestuingurua sortu baitute. Gizartearen esku-hartze era desberdinak bilatzeko beharrak, komunitatean jartzen du arreta hautabide garrantzitsua izan daitekeelako Ongizate Estatuak babesten ez dituen arloei erantzuna emateko. Artikulu honek, Europako herri desberdinetako (Erresuma Batua, Herbehereak eta Italia) gobernuetatik burututako politikak aztertzen ditu. Zentzu berean, hiru politika desberdin hauen gogoeta nagusiak adierazten ditu komunitatearen funtzioa sustatuz, ongizate sistemaren iraunkortasuna bermatu ahal izateko.
\end{abstract}

\section{GAKO-HITZAK:}

Komunitatea, aktibatze komunitarioa, ekimen komunitarioak, Ongizate Estatua, Gizarte Politika.
La crisis financiera iniciada en 2008, además de las diversas transformaciones sociales que se han venido sucediendo en las estructuras familiares y el mercado laboral, junto con la existencia de nuevos riesgos sociales, han generado un contexto de incertidumbre en el que se cuestiona la capacidad del Estado de Bienestar para hacer frente a esta situación. La necesidad de buscar fórmulas de intervención social más eficientes y efectivas hace de la comunidad una alternativa de gran relevancia para reforzar y cubrir así aquellos espacios a los que el Estado de Bienestar no puede llegar. El presente artículo analiza y expone las principales reflexiones elaboradas a raíz de tres propuestas políticas estatales de activación comunitaria llevadas a cabo desde los gobiernos de diferentes países europeos (Reino Unido, Países Bajos e Italia) que ponen en valor la función de la comunidad en los sistemas de bienestar para garantizar así su sostenibilidad.

\section{PALABRAS ClaVE:}

Comunidad, activación comunitaria, iniciativas comunitarias, Estado de Bienestar, política social. 


\section{Introducción}

Históricamente, el modelo guipuzcoano de servicios sociales se ha caracterizado por la estrecha vinculación del sector público con el tercer sector y la iniciativa privada. No obstante, factores tales como el envejecimiento de la población, la presión a la que está sometido el Estado de Bienestar, el contexto económico de crisis y otra serie de cambios sociales han generado un contexto en el que se hace necesaria la reconfiguración de los sistemas de bienestar. Es por ello por lo que en los últimos años se ha prestado especial atención a diferentes iniciativas sin ánimo de lucro haciendo hincapié en los vínculos comunitarios y relacionales en el desarrollo de políticas sociales como complemento y refuerzo de la Administración Pública de cara a garantizar su sostenibilidad.

Estas iniciativas se han ido materializando en los países de nuestro entorno mediante la creación y aplicación de programas que tienen como fin último impulsar la creación de redes de solidaridad vecinal y el desarrollo de modelos de voluntariado de proximidad. De tal manera, el objetivo del presente artículo es reflexionar acerca de las principales iniciativas o estrategias políticas propuestas en el contexto europeo que pongan en valor la función de la comunidad en los sistemas de bienestar tal y como se ha hecho para el proyecto de investigación desarrollado conjuntamente con la Diputación Foral de Gipuzkoa.

De tal manera, en un primer momento se hace una revisión del Estado de Bienestar en Europa, centrándose en las diferentes formas de provisión de bienestar en diferentes países, para abordar el caso del Estado español y sus principales retos en la actualidad. El artículo también estudia el concepto de comunidad, el cual se entiende en términos de territorialidad, y da paso al análisis de diferentes modelos de participación en el sistema de bienestar, como por ejemplo la intervención comunitaria, el enfoque comunitario de los servicios sociales, la corriente política comunitarista y la gestión de los bienes comunes. A partir de estas realidades, cabe preguntarse sobre la aplicabilidad de esta reflexión en nuestros entornos más cercanos.

\section{Crisis del Estado de Bienestar}

El Estado de Bienestar europeo, definido como "un conjunto de instituciones estatales proveedoras de Políticas Sociales dirigidas a la mejora de las condiciones de vida, a la protección de la ciudadanía y a procurar su igualdad ante los riesgos derivados del ciclo vital y de oportunidades" (Moreno et al., 2014: 9), se va constituyendo durante el periodo posterior a la Segunda Guerra Mundial (1939-1945) ante la necesidad de paliar las desigualdades existentes en la sociedad mediante prestaciones sociales orientadas a aquellos colectivos más desfavorecidos. Más concretamente, autores como
Del Pino y Rubio (2016) sostienen que existen diferentes teorías explicativas del surgimiento de los Estados de Bienestar, entre las que destacan tres: las teorías funcionalistas, que defienden que los Estados de Bienestar son resultado de la industrialización; la teoría de los recursos de poder, según la cual fueron el resultado del conflicto entre las clases sociales; y, finalmente, la teoría institucionalista, que sostiene que los estados centralizados con grandes instituciones burocratizadas eran el caldo de cultivo perfecto para el desarrollo de los Estados de Bienestar.

De tal manera, el desarrollo del Estado de Bienestar queda ligado a diferentes fases, dentro de las cuales se distinguen las siguientes (Taylor Gooby, 2000; Moreno, 2012): la Edad de Oro (1945-1975), conocida como la fase del máximo apogeo del capitalismo del bienestar europeo; la Edad de Plata (19672007), en la que fueron transformándose frente a la presión ejercida por las corrientes neoliberales que procuraban su desmantelamiento; y la Edad de Bronce (2007-actualidad), caracterizada por la crisis financiera iniciada en 2008, la cual marcó un antes y un después en el proceso de desarrollo del Estado de Bienestar poniendo en cuestión su devenir.

Esta crisis económica, además de las transformaciones sociales, el surgimiento de nuevos riesgos sociales derivados de la transición de la sociedad moderna a la etapa posmoderna y la cada vez más determinante globalización económica, han desencadenado un contexto de incertidumbre en el que el Estado de Bienestar ha sufrido un claro deterioro y, por ende, se ha visto expuesto a un retroceso en su expansión, haciendo necesaria su reconfiguración.

\section{La redefinición de la función de la comunidad como estrategia para la mejora de los sistemas de bienestar}

En los últimos años, en gran medida por la presión que ha ejercido un contexto económico adverso y por la necesidad de buscar fórmulas más efectivas y sobre todo eficientes de intervenir en la sociedad, está aumentando el interés por las políticas y los mecanismos de acción comunitaria. Tanto a nivel estatal como a nivel regional e incluso municipal, se percibe un reverdecimiento del interés por la comunidad y por formas de intervenir más comunitarias (Rebollo y Morales, 2013).

\subsection{Que se entiende por comunidad}

Desde una perspectiva operativa, partiremos de que, en su conceptualización, el término comunidad refiere a su concepción geográfica. De tal manera, la comunidad se puede entender en términos de territorialidad (Moulaert, 2010) y a partir de las divisiones administrativas que se establecen para su configuración. Por lo tanto, será el barrio o el pueblo, 
en su caso, lo que se establece como comunidad. En este sentido "el barrio se ha conceptualizado como el espacio intermedio entre lo rural y lo urbano, entre lo doméstico y la metrópoli, entre lo privado y lo institucional (Castro-Coma y Martí-Costa, 2016: 135), por lo que "se utiliza el concepto en un sentido intuitivo, apelando al imaginario que acostumbran a compartir los/as operadores/as de la acción comunitaria y que nos remite a la idea de 'actores que se encuentran en un territorio delimitado', en cierto modo asimilable a 'barrio', o a 'territorio' (Rebollo y Morales, 2013: 304).

Por lo tanto, si bien desde una perspectiva operativa la comunidad puede ser entendida como “espacializada" (Moulaert, 2010), y se acota o delimita a un entorno geográfico concreto, será el sistema de relaciones sociales que se dan en un espacio definido lo que forme la comunidad (Kisnerman, 1990). Siguiendo a Sennet (2000), el lugar es geografía, una localización de la política; la comunidad evoca las dimensiones sociales y personales del lugar. $Y$ hay que recordar, como hace Kisnerman (1990), que la delimitación geográfica es relativa, ya que no hay población herméticamente cerrada. En cualquier caso, la significación de comunidad necesita de una mirada más amplia que la que aquí estamos empleando, circunscrita a su elemento territorial.

\subsection{La comunidad en el sistema de bienestar}

En torno a la provisión de bienestar, las clasificaciones establecidas en los estudios clásicos, como los de Esping-Andersen (1993), identifican como principales fuentes de provisión de bienestar el Estado, el mercado y la familia. Estas fuentes de provisión conforman el denominado welfare triangle (Esping-Andersen, 1993). No obstante, actualmente podríamos aludir a un cuarto subsistema, en el que se introduce o bien a la iniciativa social o a la comunidad como una fuente de provisión del bienestar. Como ejemplo se proponen a continuación las clasificaciones propuestas por Daly y Lewis (2000), Razavi (2007), Maino (2015) y Fantova (2014):

- Las clasificaciones propuestas por Daly y Lewis (2000) y Razavi (2007) se centran en la incorporación de la perspectiva de género al estudio de los sistemas de bienestar. Teniendo como eje central para el estudio de los Estados de Bienestar el valor del cuidado, Daly y Lewis (2000) proponen ampliar el triángulo e incluir como categorías de análisis de la provisión del bienestar al Estado, al mercado, a la familia y al sector voluntario.

- Desde la misma perspectiva, Razavi (2007) propone el "diamante del cuidado", en el que al clásico esquema del welfare triangle se añade el sector sin ánimo de lucro. Tanto el sector voluntario propuesto por Daly y Lewis (2000) como el sector sin ánimo de lucro de Razavi (2007) son un agregado heterogéneo que puede abarcar desde la comunidad, hasta al sector voluntario o no lucrativo (Tercer Sector) en la provisión del bienestar.

- Las clasificaciones de Maino (2015) y Fantova (2014), en cambio, no integran en sus esquemas a la familia. Cuestión que desde la perspectiva de los cuidados no sería concebible por el importantísimo papel que cumple y que, por tanto, se le otorga en las clasificaciones de Daly y Lewis (2000) y la de Razavi (2007). No obstante, dan cuenta de otra forma de concebir los sistemas de bienestar, en la que a la familia no se le otorga formalmente un papel determinante. Es una visión orientada a sistemas de bienestar que superen el sesgo familista y que se organizan, de tal modo, sin delegar funciones en ella. Cuestión que resulta especialmente interesante en sistemas de carácter familiarista como los mediterráneos. De esta forma, la propuesta de Maino (2015) integra en su esquema Estado, mercado, Tercer Sector y la ciudadanía, mostrando así una clara voluntad de integrar a la sociedad civil en el esquema del bienestar. Pese a que no menciona la comunidad, habla tanto de sociedad civil organizada (Tercer Sector) como de la sociedad civil no organizada (ciudadanía).

- Fantova (2014), por su parte, amplía el planteamiento especificando cuáles son los bienes que gestiona cada uno de los subsistemas y las lógicas por las que se rigen. El autor alude como fuentes de provisión a la iniciativa social, al Estado, al mercado y a la comunidad. En lugar de ciudadanía, el autor se refiere al sistema que representa la comunidad, lo cual puede ayudar a acotar la realidad a la que se hace referencia y, desde una perspectiva operativa, puede resultar más concreto que el concepto de ciudadanía, que resulta, por lo general, más abstracto y difuso. Esta propuesta establece la separación entre comunidad y sector voluntario o Tercer Sector para hacer énfasis en que la comunidad está formada por relaciones de carácter más informal que las que se dan, por ejemplo, en el Tercer Sector. Esta separación resulta también pertinente para establecer la distinción entre el conglomerado que abarca todo el sector voluntario o sin ánimo de lucro.

Dependiendo de la perspectiva desde la que se proponga el esquema y el objetivo para el que se diseñe, emplear una u otra categorización puede, o no, facilitar la labor de análisis de los sistemas de protección social. Además, es necesario tener en cuenta que no existe equivalencia funcional (Fantova, 2014) entre los diferentes servicios o dimensiones del bienestar que provee cada subsistema. La función de cada uno de los sistemas es, por tanto, igual de trascendental que diferenciada. De esta forma, se entiende que la función de uno de los sistemas no puede ser sustituida por la función de otro. La comunidad puede ser efectiva, puede ser participativa y, así, integrada en mayor medida en los sistemas de protección, pero las acciones que se 
llevan a cabo en esta dimensión no deberían originar la sustitución de servicios que forman parte y son, de tal forma, competencia de otros subsistemas. En esta línea, la comunidad respondería, en su función, a las lógicas que exclusivamente atañen a la vida social y relacional de quienes la constituyen.

\subsection{Diferentes niveles de integración de la comunidad en los sistemas de bienestar}

La forma en la que actualmente la comunidad participa en la provisión de bienestar parece no estar teniendo un impacto considerable. Desde la intervención social, la perspectiva comunitaria ha sido, como se expone a continuación, relegada en detrimento de la perspectiva individual (o grupal en cualquier caso), teniendo actualmente muy poca presencia en la práctica y en el imaginario de los/as profesionales de este ámbito. Esta es, por tanto, una de las principales razones por las que la comunidad puede no estar teniendo un papel trascendental en el objeto de hacer efectivo el bienestar en la ciudadanía. Por lo tanto, uno de los retos principales implica resolver cómo implementar políticas de activación comunitaria.

En el contexto de crisis del Estado de Bienestar, se vienen trabajando teorías y propuestas que enfatizan la necesidad de otorgar una función más determinante a la comunidad como sistema garante de la protección social. Las cuatro perspectivas que se exponen a continuación, si bien pueden ser complementarias y no rivalizan entre sí, suponen diferentes grados de integración de la comunidad en los sistemas de bienestar.

\subsubsection{La intervención comunitaria como la clásica forma de relación entre institución y comunidad}

La metodología clásica de la intervención con la comunidad ofrece un marco de referencia para entender la forma en la que es posible llevar a cabo un proceso de trabajo comunitario. El concepto de desarrollo e intervención comunitaria es planteado como uno de los tres niveles de intervención (junto con el individual y el grupal) de las disciplinas del ámbito social y considerado como referente metodológico fundamental en aras de garantizar el bienestar y la protección social (Viscarret, 2007).

Conviene destacar que, pese a ser un referente metodológico importante, no es una práctica extendida y toda la labor de los/as profesionales de la intervención social gira en torno al trabajo de caso o la intervención individual. Ramos (2000: 199) afirma que, a este respecto, la modernización lo comunitario y, en términos de prácticas profesionales, “lo arrasó”.

Pese a que estas prácticas no constituyen una forma habitual de intervención en el contexto español, su fundamentación teórica sirve de base para entender la forma en la que se pueden llevar a cabo procesos comunitarios que deriven en un nuevo marco de relación entre instituciones y comunidad. Partiendo de la teoría de la intervención comunitaria, se exponen a continuación una serie de principios básicos de la intervención:

\section{- Principio 1. Participación}

La condición básica a la hora de desarrollar un proceso de trabajo comunitario es que las personas y los grupos aseguren su participación. Siguiendo a Seoane y Rial (2001), el desarrollo comunitario y la participación son procesos inseparables en los que la acción social no se desarrolla en la comunidad, sino de y desde ésta, de tal forma que la población, para la constitución y consolidación de la comunidad, asume responsabilidades, toma decisiones y es responsable de los resultados y de las consecuencias de su evolución.

\section{- Principio 2. Organización}

El principio operativo fundamental del trabajo con las comunidades es la organización de individuos y grupos, así como la generación de redes. Se trata de una organización con intencionalidad, que tiene como objetivo hacer frente colectivamente a problemas, necesidades o intereses colectivos (Rebollo y Morales, 2013). De esta forma, la intervención comunitaria "se trataría de una práctica organizativa que realizan los profesionales del ámbito de la intervención social y en torno a ciertos objetivos colectivos" (Barbero, 2010: 65).

Dependiendo del nivel de organización y estructuración de las redes y en función del carácter formal o informal de estas, se determinará su funcionalidad para abarcar la protección social. Por tanto, dependiendo de las características de las redes, se podrán abarcar unas u otras cuestiones que atañen al desarrollo de la comunidad. Se trata de que en cada tipo de red se intervenga de diferente forma y en base a objetivos diferenciados.

- Principio 3. Factores estructurales de la comunidad: territorio, población, demandas y recursos

Siguiendo el planteamiento realizado por Marchioni (1999), los factores que determinan la intervención con la comunidad son: el territorio, la población, las demandas y los recursos. En primer lugar, las características físicas del entorno marcan una serie de posibilidades y de limitaciones que han de ser tenidas en cuenta. De esta forma, la segregación de las personas, los obstáculos físicos e incluso el clima suponen factores a considerar. Respecto a la población, las características de las personas que habitan en ella influyen en el proceso de intervención comunitaria. Su influencia no responde solo a 
una cuestión sociodemográfica, también cabría atender a los valores, hábitos y costumbres en los que estos basan su vida en la comunidad.

En esta línea, se considera que las personas, los grupos y las comunidades cuentan con una serie de recursos que son claves en el proceso de intervención. La identificación y activación de estos recursos será, por tanto, el primer paso a dar en el proceso de la intervención. A este respecto, las redes y el capital social existente constituyen recursos fundamentales sobre los que apoyarse a lo largo de un proceso de intervención en la dimensión comunitaria.

Además de estos principios básicos, se ha de tener en cuenta que los programas, proyectos, intervenciones y las demás formas de acción social que se deseen implementar en las comunidades deben responder a necesidades y demandas sentidas, latentes y manifiestas en ellas, y no únicamente a necesidades demandadas por la institución implicada. Con frecuencia se llevan a cabo procesos que apenas responden a necesidades 0 demandas explicitadas por la comunidad, de manera que el impacto que puedan tener se reduce al plano político. Como señala Marchioni (1999) el diagnóstico de las necesidades no es patrimonio únicamente de los/as técnicos/as y de los/as políticos/as, sino, también, de las personas que habitan en la comunidad. La población, por tanto, debería tomar parte en el proceso de identificación de los problemas y, a partir de ahí, formular una serie de demandas acordes con sus necesidades. Si bien los procesos participativos alargan las intervenciones, los resultados serán fruto de un trabajo consensuado y compartido entre instituciones y comunidad.

\subsubsection{De la intervención comunitaria hacia el "enfoque comunitario" de los servicios sociales como base para la redefinición de la función de la comunidad en los sistemas de bienestar}

El enfoque comunitario de los servicios sociales es a menudo entendido como una política de desinstitucionalización o de prestación de servicios en el entorno cercano o comunitario, ciertamente ligado al ámbito de la dependencia. No obstante, actualmente se plantea como un enfoque que, además de dirigirse a proveer servicios desde la dimensión comunitaria y a ofrecer una atención centrada en la persona en su entorno, busca favorecer la integración y la interacción de los sujetos con el medio.

Cabe destacar que, prácticamente desde los inicios de este modelo, ya era criticado por una cuestión que todavía hoy parece no haberse resuelto, ya que presenta un problema potencial al ser la familia el eje principal sobre el que se apoyan las políticas basadas en la comunidad, recayendo el peso mayormente sobre las mujeres.

La perspectiva comunitaria se dirige a fortalecer los vínculos primarios e, igualmente, a promover la extensión de relaciones entre personas, grupos y colectivos con aparentemente menor vinculación. Vínculos que en su unión son fuente de implicación, desarrollo y proyección comunitaria. Así, la perspectiva comunitaria es un conjunto de:

$$
\begin{aligned}
& \text { “Enfoques o aproximaciones que subrayan la } \\
& \text { necesidad de que las políticas sociales públicas } \\
& \text { o formales no desincentiven (sino que más } \\
& \text { bien promuevan) las capacidades, los recursos, } \\
& \text { vínculos y procesos que de manera más o menos } \\
& \text { natural o primaria existen en el seno de las } \\
& \text { relaciones y redes familiares y comunitaria”. } \\
& \text { (Fantova, 2014: 104). }
\end{aligned}
$$

Desde el enfoque comunitario, no se trata de sustituir a las instituciones públicas sino de que estas, dentro de sus acciones y políticas, pongan un mayor énfasis en transformar situaciones colectivas o comunitarias, frente a continuar desarrollando la tradicional asistencia individualizada. De esta manera, las políticas comunitarias se entenderá que no son únicamente unas políticas destinadas a una atención de cercanía y orientada al mantenimiento de las personas en su entorno habitual, sino que abarcan o deberían abarcar toda una serie de estrategias de promoción de los vínculos comunitarios y del trabajo comunitario.

Pese a no ser un referente consolidado, en la Comunidad Autónoma Vasca (CAV) el enfoque comunitario de atención queda reconocido como modelo de atención de referencia para los servicios sociales en la Ley $12 / 2008$. El marco normativo existente en este caso es favorable al desarrollo de políticas sociales de enfoque comunitario y da por tanto un primer paso hacia la transición de modelo. No obstante, como se puede concluir del informe presentado por el Ararteko (2016) acerca de la situación de los servicios sociales de atención primaria, falta un largo recorrido para su despliegue que deberá ir dándose mediante la implementación de políticas concretas.

\subsubsection{La corriente política comunitarista; la comunidad como eje en la provisión de bienestar}

La corriente filosófica y política conocida como comunitarismo o communitarianism surge en la década de los ochenta (Naval y Sison, 2000; Fondevila, 2003), y se asienta en la década de los noventa (Velásquez, 2008). Esta corriente ha sido promovida en el contexto occidental con el objetivo de enunciar una nueva teoría; un nuevo paradigma que se asiente sobre las bases de la filosofía moral y política (Naval y Sison, 2000).

El comunitarismo, en líneas generales, se basa en el siguiente principio: “Los seres humanos no solo somos sociales por naturaleza, sino que además la sociabilidad aumenta nuestra potencialidad humana y moral" (Velásquez, 2008: 105). En este sentido, los comunitaristas constatan "la disolución del nexo social 
y de las identidades colectivas, el incremento del individualismo y, en consecuencia, la generalización de una falta de sentido" (Naval y Sison, 2000: 601). En este contexto, la promoción del sentido y la acción comunitaria sería, según se postula, la fuerza garante del desarrollo social de los individuos.

La corriente comunitarista nace de la crítica a la corriente neoliberal y trata de atender los problemas que surgen en el seno de las sociedades postindustriales, apelando a los valores propios del sentido comunitario. En líneas generales, los planteamientos comunitaristas proponen recuperar parte de los valores que operaban en el pasado. Esto es, volver a formas más tradicionales de relación.

No obstante, sostener la perspectiva comunitarista, en la sociedad actual implica atender a las diferentes tendencias y corrientes que se imponen para su deslegitimación. Tendencias y corrientes de pensamiento que, en líneas generales, promulgan la individualidad en detrimento del sentido comunal. A este respecto, convendría atender a cómo conseguir la integración de las comunidades y afirmar su identidad, sin negar la diversidad y especificidad de los diversos componentes (Velásquez, 2008). El reto, en las sociedades complejas y diversificadas, es, por tanto, lograr un tipo de comunidad donde se equilibren la unidad y la diversidad. Lograr un equilibrio en un contexto de individualidad donde, en contraparte, tampoco resultaría tratar de retornar a la comunidad tradicional (Gómez, 2000).

Si bien es cierto que cada comunidad tiene su identidad propia, dicha identidad no tiene que ser necesariamente compartida por todos los miembros que la integran. En este sentido, Gómez (2000) señala que lo fundamental es la participación para el logro de un consenso. Consenso en el que cada sujeto logra, en su diversidad, reconocerse e identificarse con los demás. Consenso en el que los derechos del individuo deben ser reivindicados, al igual que las responsabilidades que le repercuten. En este sentido, Velásquez (2008) considera que en el comunitarismo se reivindica la afirmación de los deberes sobre los derechos. Naval y Sison (2000), por su parte, señalan que, además de la búsqueda del equilibrio entre individualidad y comunidad, se debe concretar cuál ha de ser el papel de las instituciones que alimentan valores morales dentro de las comunidades.

Como señala Gómez (2000), el vínculo en la comunidad se entiende como natural, mientras que en la asociación se basa en la convención. Si a menudo se han planteado estos conceptos como contrapuestos, se pueden entender, por el contrario, como complementarios, puesto que a través de las relaciones que se dan en las asociaciones también se generan elementos comunitarios. Esta puede suponer para el comunitarismo una clave importante, ya que en la actualidad el vínculo natural puede resultar ciertamente anacrónico, pese a que pueda ser igualmente reivindicado. La construcción de comunidad por medio de la asociación puede ser, de este modo, una clave teórica importante que sirva de argumento y orientación para la implementación de políticas de activación comunitaria.

\subsubsection{Los comunes o la gestión común de los bienes}

Históricamente, los comunes, concepto utilizado para nombrar la tierra, los bosques y las aguas de pesca comunes pero que hoy abarca más dimensiones, se han impuesto a la propiedad privada. A lo largo de varios siglos, como consecuencia de los cercamientos (enclosure) y desamortizaciones, muchas tierras fueron pasando de la propiedad comunitaria hacia la estatal o privada. Como consecuencia también de un fenómeno central en el propio desarrollo de la conciencia moderna, los bienes comunes fueron eliminados como categoría político-cultural dotada de dignidad constitucional (Mattei, 2013: 60).

Este concepto abarca una enorme cantidad de realidades y existen diversas formas de clasificarlos que pueden ayudar a comprender la naturaleza versátil del concepto. Siguiendo a Hess y Ostrom (2016), los bienes han sido clasificados utilizando la lógica rivalidad/no rivalidad, cuando su uso por parte de un individuo reduce su disponibilidad por parte de los demás; y exclusión/no exclusión, cuando se puede excluir o impedir a los individuos su uso. De esta manera, los bienes podrían agruparse en cuatro grandes grupos: públicos, comunes, clubes y privados.

Dentro de los comunes, estos pueden ser caracterizados de muy diversas formas que van más allá del clásico uso común de los pastizales, que pueden aportar al campo de las políticas sociales una perspectiva desde la que los servicios sociales pueden ser entendidos como un bien común o asociativo. Es especialmente interesante desde la perspectiva de las políticas sociales el concepto de comunes sociales. Es el que propone Mestrum (2016), con el objetivo de hacer partícipe a la ciudadanía de la creación, implementación y regulación de la protección social. La autora plantea entender la protección social como un bien común y no como un derecho ligado únicamente a la relación con el Estado.

Como afirman Nogués y García (2016: 4), el común es un concepto en construcción, que reclama del pasado premoderno, precapitalista, algunas fórmulas e ideas que ya fueron cuestionadas a la modernidad. Supone la ruptura de los vínculos, reconociendo el valor de formas tradicionales o premodernas de relación y el papel que las comunidades pueden jugar en la actualidad. Gutiérrez-Rubí (2012) señala que se presenta como la recuperación de los valores que aproximen a la persona a la cooperación más que a la competitividad. En la actualidad "el procomún se abre paso como una de las corrientes de pensamiento (y práctica personal) que podría iluminar sendas diferentes de los caminos trazados 
hasta ahora” (Gutiérrez-Rubí, 2012: 10). Forma parte de un discurso que muestra la voluntad de relacionarse y gestionar los bienes de forma diferente e innovadora frente a la actual crisis.

Desde esta visión se plantea una nueva forma de entender lo público, más allá de la propiedad estatal de un bien o servicio, acercándolo a la ciudadanía y dotándola de mayor responsabilidad. Lo público no es solo algo entendido como titularidad del Estado, sino como algo que debe ser cogestionado o compartido. Hablar de bienes comunes supone "asumir un privilegiado punto de vista más holístico para analizar cómo puede gestionarse mejor un recurso" (Bollier, 2016: 52). Siguiendo a Zubero, “la perspectiva de los bienes comunes, del procomún, es una oportunidad para volver a pensar la autonomía y la iniciativa de los individuos sin caer en el administrativismo y la burocratización, pero sin abandonarlas en manos del mercado y su lógica competitiva. Nos permite volver a pensar la sociedad como un proyecto relacional, alejado de cualquier forma de comunitarismo tradicional, pero fundado en la construcción cooperativa por parte de individuos asociados libremente" (Zubero, 2012: 40).

En definitiva, los bienes comunes "no son un manifiesto, una ideología, una expresión de moda, sino más bien un marco flexible para expresar la rica productividad de las comunidades sociales amenazadas por los cercamientos del mercado" (Bollier, 2016: 63), que indudablemente ofrecen un nuevo marco para repensar la función de la comunidad en el sistema de bienestar.

\section{Propuestas políticas estatales de activación comunitaria}

Las tres estrategias estatales de activación comunitaria llevadas a cabo desde las políticas públicas y los gobiernos de diferentes países europeos son: la Big Society británica, la Sociedad Participativa holandesa y el Secondo Welfare italiano.

\subsection{La Big Society británica}

La Big Society británica se considera como paradigma estratégico-estatal de activación comunitaria de referencia en la política europea. Este planteamiento surge en el año 2010 como propuesta que desarrolla la coalición liberalconservadora inglesa para procurar la transición del Big Government hacia la Big Society. En otras palabras, la propuesta se dirige a descentralizar, trasladando poder, responsabilidad y capacidad de decisión, desde el Estado hacia el nivel local del Gobierno (Evans, 2011). Tal transición implica un engrosamiento del sistema social o comunitario y, en consecuencia, el desmedro del aparato público.

La principal propuesta que se plantea desde la Big Society es que las comunidades, por medio de los niveles de gobierno más locales o descentralizados, asuman mayor control sobre sus presupuestos, sobre la gestión de ciertos bienes y servicios, y que, además, sean los miembros de estas quienes decidan dónde invertir en su propio entorno. Esta propuesta de descentralización, definida como localismo desde la política británica, refiere devolución de poder, funciones o recursos desde el control centralizado hacia los gestores, tales como instituciones locales o comunidades (Evans, Marsh y Stoker, 2013).

En este sentido, el tejido asociativo, las organizaciones voluntarias, el Tercer Sector o el cooperativismo cobran especial relevancia, siendo necesario, por ende, el empoderamiento de las propias comunidades. De tal manera, el planteamiento se concreta a partir de las tres grandes estrategias identificadas: el empoderamiento de la comunidad, la promoción de la acción social y la extensión de los servicios públicos (Lowndes y Pratchett, 2012: 30).

La propuesta de Big Society, además de suponer un cambio de funcionamiento a nivel institucional, también implica promover una transformación en los valores de nuestras sociedades con el objetivo último de generar en los individuos y grupos la necesidad sentida de participar en la vida cívica. Tal y como Evans (2011) argumenta, la Big Society tiene que promover la existencia de una ciudadanía activa y poner empeño en alimentar el sentido de comunidad, el de ciudadanía y el de deber cívico.

Atendiendo a lo expuesto en el informe elaborado por Civil Exchange (2015), si bien el objetivo propuesto e integrado en el discurso de la Big Society era unir a la sociedad, tras cinco años de la puesta en marcha del proyecto, diversos datos muestran una sociedad más dividida en la que, además, el poder no ha sido repartido ni devuelto hacia las comunidades. La actitud de descrédito por parte de los participantes respecto a su capacidad para influir en el ámbito local y la constatación del desencanto generalizado hacia la política según se expone en los datos del informe, dan fe de los inconvenientes derivados de la experiencia propuesta.

Otra de las carencias identificadas que han imposibilitado su mayor expansión ha sido que los planes propuestos han respondido más a necesidades u objetivos del Gobierno que a los propios problemas y necesidades reales de las comunidades o personas (Smith, 2011). Este hecho ha reforzado la gran reticencia con la que organizaciones y comunidades habían recibido el planteamiento. En este sentido, ha sido patente la incapacidad del Gobierno para reorientar la visión negativa que se ha generado, lo que ha supuesto la imposibilidad de retomar el desarrollo del planteamiento.

Bien es cierto que también pueden desprenderse aprendizajes positivos de esta experiencia. En 
este sentido, es de destacar la gran capacidad de movilización de recursos que algunas de las comunidades han mostrado, pudiendo así llevar a cabo diferentes experiencias a nivel local; además del crecimiento del voluntariado y apego social del colectivo de los/as más jóvenes, ya que el voluntariado en la franja de edad entre los 16 y 24 años se ha duplicado desde el año 2005 (Civil Change, 2015).

En esta línea, se abre el debate sobre la capacidad de ciertas comunidades, especialmente aquellas más deprimidas o con menos recursos, para poder tomar las riendas y asumir las responsabilidades que la Big Society requiere (Wyler, 2011). A este respecto, autores como Chanan y Miller (2011) indican que la verdadera función de las comunidades no reside ni en tomar el control de los servicios públicos ni en sustituir las responsabilidades del Estado, sino que deben complementar y coproducir los servicios públicos.

Aunque el planteamiento de la Big Society haya sido considerado por muchos análisis como una forma relativamente novedosa y políticamente correcta de presentar políticas neoliberales de ajuste estructural (Fantova, 2014), es necesario tener en cuenta que, para su correcto y eficaz funcionamiento, los mecanismos que se desplieguen para implementar estas estrategias suponen un gran reto tanto técnico como teórico. Uno de los aspectos principales a tener en cuenta para el correcto funcionamiento de este planteamiento hace referencia a la necesaria transformación tanto del Estado como de los valores de la sociedad. En este sentido, y para que ambas figuras puedan ajustarse al cumplimiento del objetivo del paradigma que se viene presentando, es necesario un acercamiento entre ambos que posibilite su trabajo conjunto.

\subsection{La Sociedad Participativa holandesa}

El discurso político que el rey Guillermo de Holanda llevó a cabo en el año 2013 desencadenó un arduo debate acerca de uno de los pilares más característicos y básicos de los estados europeos: el Estado de Bienestar. El discurso del monarca apostaba por la transformación del Estado de Bienestar holandés por una sociedad participativa.

Esta propuesta de transformación se enmarca dentro de la percepción de que el Estado de Bienestar holandés, caracterizado por el incremento en el gasto público, el aumento de la esperanza de vida y el envejecimiento demográfico de la población, es insostenible sin la realización de reformas sustanciales que posibiliten su continuidad.

Es bajo este contexto donde surge el paradigma de Sociedad Participativa, que defiende y tiene como base los conceptos de participación e implicación ciudadana de manera que la ciudadanía asuma una mayor responsabilidad, no solo sobre su propio bienestar, sino también sobre la sociedad en su conjunto. En otras palabras, podría decirse que la ciudadanía debe mostrarse como sujeto activo para cumplir con su obligación de beneficiar a la sociedad y cumplir así con los prerrequisitos para convertirse en buen ciudadano/a (Delsen, 2016; Schinkel, 2010). De tal manera, sus objetivos principales son, por un lado, reforzar o empoderar la sociedad y, por el otro, demoler los derechos sociales y sustituirlos por la responsabilidad que cada una de las personas debe asumir ante los diferentes riesgos sociales.

Una de las claves de cambio hacia un modelo de sociedad participativa pasa por impulsar una transformación tanto en la actitud de los/as beneficiarios/as de las ayudas públicas como en su carácter, puesto que estas dejarían de ser consideradas o percibidas como derechos, pasando a convertirse en ayudas que tienen como finalidad última ayudar a que la persona salga de la situación de necesidad en la que se encuentra. De tal manera, los/as beneficiarios/as, para no perder la prestación correspondiente, contarían con determinadas obligaciones que tendrían que cumplir.

La actitud del Estado en la práctica de este paradigma es otra de las claves a la hora de potenciar el modelo de Sociedad Participativa del que se viene hablando. En este sentido, es necesario que el Estado se acerque a la ciudadanía abriéndole sus puertas y facilitando su participación activa en aquellos espacios de gobernanza que hasta ahora tenían cerrados (Cornwall, 2002; Newman y Tonkens, 2011).

A pesar de las conclusiones obtenidas tras la puesta en práctica del paradigma de la Sociedad Participativa, bien es cierto que desde que esta propuesta vio la luz en septiembre de 2013 ha sido objeto de diferentes críticas que guardan estrecha relación con el sentimiento de abandono que la sociedad, especialmente los colectivos de mayor vulnerabilidad, pueden llegar a presentar por parte del Estado (Koster, 2014). Otra de las críticas recibidas hace referencia a la desregularización que en el anterior párrafo se comentaba, ya que, a pesar de ella, el Estado sigue jugando un papel importante, tratando de regular y formalizar las prácticas informales (Koster, 2014).

A pesar de que la Sociedad Participativa sea considerada por algunos como la versión mejorada de la sociedad holandesa (Koster, 2014) y las conclusiones obtenidas en sus prácticas demuestren un buen funcionamiento, lo cierto es que resulta complejo visualizar la obligatoriedad de participación en aquellos grupos o barrios más vulnerables de la sociedad que no dispongan de las herramientas necesarias o suficientes para cumplir con este requisito. En este sentido, es importante tener en cuenta las consecuencias que este modelo puede llegar a generar en aquellos sujetos "no participativos” (aislamiento, dependencia, etc.), consecuencias que, a largo plazo, podrían llevar a la reproducción de las características del sistema de bienestar que se ha pretendido modificar. 


\subsection{El Secondo Welfare italiano}

El debate público sobre la insuficiencia del Estado de Bienestar italiano ha ido en aumento desde el año 2008. A pesar de las múltiples reformas que se han llevado a cabo en el país a lo largo de estas dos últimas décadas, el modelo de bienestar Italiano sigue caracterizándose por, en su postura familista, apostar por las estructuras familiares que, en el rol actual que desempeñan, se han quedado obsoletas. De este modo, en el contexto italiano se constata un gasto social desequilibrado, el envejecimiento de la población, el desempleo y las malas condiciones laborales, y unos núcleos familiares cada vez más reducidos (Maino, 2015).

En este sentido, aunque el Estado de Bienestar italiano continúe amortiguando tales efectos, es razonable plantearse si podrá continuar haciéndolo en un futuro. Es precisamente bajo este contexto donde surge y se plantea la necesidad de replantear las políticas familiares y de cuidado propias del país.

La necesidad del cambio de paradigma deviene de la creciente brecha que se abre entre necesidades y demandas de la ciudadanía y las respuestas que los diferentes servicios y programas están generando. En otras palabras, se exige una mayor velocidad de adaptación de la oferta a la diversificación de la demanda. Sin embargo, la falta de adecuación de la oferta organizativa del Estado de Bienestar supone la búsqueda de nuevos modelos de organización que complementen los vigentes. Además, las todavía imperantes restricciones financieras hacen impensable la expansión del Estado de Bienestar público. Es decir, no es cuestión de sustituir el gasto público por el gasto privado, sino de movilizar recursos adicionales para necesidades y expectativas crecientes.

Es bajo este contexto de crisis del Estado de Bienestar donde surge el denominado Secondo Welfare como propuesta referente para el diseño de políticas sociales de activación comunitaria y como una alternativa para dar soporte y promover un cambio de paradigma que lleve a la renovación del Estado de Bienestar, frente a las amenazas de su desmantelamiento. Esta propuesta italiana enfatiza el papel de la sociedad civil y el del tercer sector como provisores de bienestar. Así, características tales como la descentralización y el localismo también juegan un papel primordial. Precisamente, una de las vías que esta estrategia persigue defiende la desmercantilización del bienestar apostando, según se argumenta, por una sociedad civil dotada de reflexividad relacional que está constituida por sujetos de corte comunitario y societario como son las familias, las organizaciones del Tercer Sector y los demás entes locales (Fantova, 2014).

La denominación del Secondo Welfare o "segundo welfare" no se refiere a la creación de un nuevo sistema en términos de sustitución del primero, sino a la creación de un sistema paralelo que sirva de complemento. En otras palabras, se basa en un segundo nivel de provisión que refuerza al ya existente, de manera que los servicios que surjan de este vendrían a complementar espacios que el primer welfare no llega a cubrir. Así, los pilares del Estado de Bienestar (educación, sanidad, seguridad social y servicios sociales) se entienden como competencia del primer welfare, por lo que los servicios troncales deben ser provistos en este nivel. Mientras que todos aquellos riesgos y necesidades que en la actualidad se presentan, y que no pueden ser abordados en el primer welfare, son susceptibles de ser resueltos en el segundo welfare. Por ejemplo, en el caso de la dependencia, el primer welfare cumpliría con la necesidad de las pensiones, la asistencia sanitaria, alojamiento o servicios domiciliarios, entre otros, mientras que el segundo welfare complementaría esas prestaciones con servicios de asistencia de carácter menos vital o fundamental, como los programas de apoyo y acompañamiento, ocio, formación, etc.

Se trata de un modelo de gobernabilidad vertebrada alrededor de la asociación público-privada, tanto en la dimensión social como en la dimensión del ánimo de lucro. Es decir, si el protagonista único del primer welfare es el Estado, en el segundo este pierde su titularidad y se le unen otra serie de protagonistas: gobiernos locales (regiones y ayuntamientos), el sector privado, el Tercer Sector y la sociedad civil (asociaciones, sindicatos, etc.) que generan dinámicas de colaboración y que contribuyen a la provisión de determinado servicio. De este modo:
“El segundo welfare surge, por tanto, de una colaboración más estrecha entre los sujetos pertenecientes a las cuatro esferas e incluso involucra a stakeholders de diversa naturaleza: empresas, sindicatos, entes bilaterales, fundaciones, aseguradoras, fondos y organizaciones del Tercer Sector”. (Maino, 2015:113)

En esta línea, la esfera pública interviene por medio de las Administraciones Locales, que son asignadas para asumir un rol central en la promoción de colaboraciones público-privadas y que, de igual modo, deben centrarse en la identificación de recursos adicionales (Maino, 2015). Este rol, por tanto, se deberá centrar en dos aspectos fundamentales: por un lado, facilitar la innovación y el surgimiento de nuevas estrategias y, por otro, garantizar los derechos sociales existentes.

Dentro del Secondo Welfare se sitúan medidas de innovación social. A saber, programas de protección e inversiones sociales con financiación no pública, que se suman y entrelazan con el primer welfare, de tal forma que lo referente al primer welfare se define como de naturaleza pública y obligatoria, y lo referente al segundo como privado, opcional y complementario. Es decir, la respuesta a los problemas parece estar basada en una combinación de programas de protección social no financiados 
por el Estado sino, más bien, proporcionados por un amplio conjunto de actores económicos y sociales conectados en red y con un fuerte arraigo territorial (Maino, 2015).

Además, conviene destacar que una de las capacidades que ofrece este esquema es la mayor flexibilidad que tienen los protagonistas que intervienen, ya que les permite superar la rigidez del welfare tradicional o primero, ya que se trabaja en un contexto más propicio para la innovación y para la puesta en marcha de iniciativas centradas, de forma más concreta, en las necesidades de cada comunidad.

En estas experiencias, se trata de que se implementen políticas y servicios orientados a mejorar las condiciones de vida de los sujetos que se constituyen en la comunidad, a partir de la ejecución de acciones de mejora promovidas desde el sector privado y desde el Tercer Sector que, en su implicación, cuentan con el apoyo institucional de la Administración Pública. En esta línea, el Secondo Welfare se constituye a través de espacios de comunicación y acuerdo que se establecen a partir de la interrelación de los citados agentes. Interrelación por la que se pretende concretar, entre ellos, pactos territoriales por los que asegurar la protección social de la ciudadanía.

En efecto, la constitución del Secondo Welfare evidencia que, por la consolidación de la crisis del Estado de Bienestar, por la falta de espacios de comunicación entre agentes implicados en este ámbito y por los impedimentos constatados para hacer efectiva la centralización de los servicios por parte de la Administración Pública, resulta necesario diseñar estrategias para repensar y asumir la transferencia del riesgo y la búsqueda de soluciones alternativas desde el ámbito de la comunidad.

\section{Discusión}

Tal y como defienden autores de referencia en el estudio de las sociedades occidentales como Bauman (2003) o Sennet (2000), se está generando, en contra de la lógica de la modernidad, cierto reverdecimiento del interés y la necesidad de comunidad. No obstante, es necesario reflexionar y preguntarse si las propias comunidades cuentan con capacidad suficiente como para hacer frente a los requerimientos o las exigencias que supondría dotarlas de mayor poder.

La sociedad actual se caracteriza sobre todo por un importante cambio de valores dentro de los cuales el individualismo ha ido adquiriendo cada vez mayor protagonismo. Además, la diversidad de intereses y de valores, a menudo contrapuestos, existentes en las sociedades postmodernas exige que la construcción de la comunidad no pueda basarse en la construcción de un "nosotros" excluyente y probablemente deba ser construido en torno a una relación de solidaridad impersonal. En este sentido, Mestrum (2016) plantea que la solidaridad orgánica y anónima puede favorecer esto y de la misma forma se deberá tener en cuenta y evitar que "las comunidades puedan convertirse en discriminatorias, sobre todo con respecto a aquellos/as que no se conforman con las normas dominantes $y$, por consiguiente, no se ajustan a ellas" (Mestrum, 2016: 35). La comunidad postmoderna no será, por tanto, "natural” sino "artificial”, construida en base a una solidaridad orgánica e impersonal.

También hay que preguntarse si realmente existe la posibilidad de que la propia ciudadanía cuente con la energía suficiente para asumir el trabajo comunitario después de su jornada laboral, de las tareas domésticas y de cuidados. Sin duda alguna, la participación en la comunidad, mediante el voluntariado $\mathrm{u}$ otra forma no remunerada, supone una inversión de tiempo y energía de la que quizás no se disponga con las exigencias que el mercado laboral actual (flexibilidad, horarios partidos, minijobs, etc.) impone. Estas características del contexto social actual hacen pensar que, a día de hoy, las comunidades no están preparadas para hacerse cargo de la gestión de servicios que supongan un alto grado de implicación o compromiso.

Por otra parte, cabe preguntarse si el interés creciente por la activación comunitaria viene promovido únicamente desde una lógica liberal que trata de adelgazar los sistemas públicos de protección social o, en contraposición, se propone como un paso adelante del Estado de Bienestar que trata de articular sistemas de protección paralelos a los existentes, es decir, si la comunidad se plantea como un sustituto o como un complemento del Estado. De las experiencias y la literatura analizadas se desprende que es un tema que suscita interés desde diversos posicionamientos ideológicos y que existen planteamientos que defienden tanto uno como otro. Hay que reconocer que los gobiernos que han impulsado las estrategias estatales de activación comunitaria expuestas lo han hecho desde una perspectiva comunitarista y, por tanto, dentro de una lógica liberal. No obstante, si bien como complemento y no como sustituto, el interés por la comunidad como fuente de provisión de bienestar también es propuesto desde lógicas socialdemócratas y socialistas.

Este uso e interés compartido e idealizado que se está haciendo de la comunidad lo convierte en un planteamiento ambiguo. Esto obliga por tanto a clarificar y definir correctamente qué es lo que se pretende con la puesta en marcha de una estrategia de activación comunitaria, porque de lo contrario puede ser interpretado como un discurso vacío, o incluso como una amenaza para el Estado de Bienestar.

En el caso de las diferentes estrategias analizadas, propuestas tal y como se ha mencionado desde lógicas liberales, se puede concluir que sus 
resultados no han sido los propuestos en sus manifestaciones programáticas. Tras unos años de su puesta en marcha, la vigencia de estas estrategias es cuestionable y pese a que no han sido abandonadas, no han tenido un impacto estable ni han sido bien recibidas ni por las comunidades ni por las organizaciones del Tercer Sector, teóricas "patas" principales sobre las que se debían desarrollar estas estrategias.

Una de las discusiones centrales es la que gira en torno al choque de legitimidades de una y otra esfera. Es decir, si el Estado tiene la legitimidad y la competencia para intervenir en el ámbito comunitario o si, por el contrario, la comunidad es la única con legitimidad para decidir qué políticas se impulsan. De esta forma, se pone en cuestión que una política de activación comunitaria pueda tener un impacto o un éxito considerable surgiendo del ámbito estatal si no cuenta con amplia aceptación social.

Son por tanto las políticas top-down, o de arriba abajo, capaces de articular estrategias comunitarias o, por el contrario, han de ser iniciativas bottom-up las que lideren esta activación. Si bien la respuesta puede ser que ambas son complementarias, hay que reconocer que de las estrategias analizadas se puede concluir que en ocasiones se dan choques de intereses, e incluso de legitimidades, entre lo que se propone a nivel institucional y lo que reclaman las asociaciones o entidades que trabajan en las comunidades.

Hay que reconocer también que en muchos casos han sido vistas como formas políticamente correctas de introducir recortes y privatizaciones (Fantova, 2014), sin haber logrado un empoderamiento comunitario efectivo. El peso del sector privado ha crecido en detrimento del sector público, lo que motiva otra de las discusiones respecto al papel que ha de jugar el sector privado en estas experiencias. Es oportuno reflexionar sobre cuál tiene que ser la función que debe cumplir, no solo en las estrategias de activación comunitaria, sino también en los sistemas de bienestar. En todas las experiencias propuestas, el mercado debía asumir teóricamente un papel importante y buscar, junto con el resto de sectores, estrategias e iniciativas innovadoras en favor del bien común. Del análisis de estas experiencias se deriva que este objetivo no ha sido verdaderamente cumplido y cabe preguntarse si es posible que las entidades privadas estén dispuestas realmente a esa búsqueda del bien común.

Pese a todas las críticas que se puedan hacer sobre las estrategias analizadas, hay que reconocer que sirven de referencia y que de ellas se desprenden una serie de aprendizajes y discusiones que pueden ser altamente interesantes desde la perspectiva de cualquier institución que pretenda poner en marcha procesos de activación comunitaria. Teniendo en cuenta las características del territorio de Gipuzkoa, se proponen a continuación tres propuestas que pueden servir de guía o de inspiración para el diseño de una estrategia de activación comunitaria en el territorio.

\subsection{Propuestas hacia la integración de la comunidad en los sistemas de bienestar}

A continuación se muestran diferentes propuestas a tener en cuenta con la finalidad de integrar a la comunidad en los sistemas de bienestar.

\subsubsection{Fortalecer y reorientar la intervención comunitaria}

Tal y como se ha mencionado anteriormente, la intervención comunitaria y las políticas de activación comunitaria ni son una práctica extendida ni forman parte del hacer cotidiano de los servicios sociales. En primer lugar será necesaria, por tanto, una redefinición de las formas de intervención social para cambiar la forma en la que las instituciones se relacionan con la comunidad. Y no únicamente atendiendo a cuestiones cuantitativas (aumentar el número de intervenciones comunitarias), sino también cualitativas (cambiar la forma y los objetivos con los que se interviene).

En este sentido, la atención primaria competente en materia comunitaria debería jugar un papel fundamental, pero tal y como se concluye en el último informe del Ararteko (2016) o en los informes del Consejo Vasco de Servicios Sociales, este nivel de atención se encuentra en una situación menguante que va en claro declive.

Unos servicios de base con escasos recursos, deficientemente coordinados y con gran dependencia económica de las diputaciones, difícilmente podrán cumplir efectivamente con el trabajo que supone reorientar su labor hacia la intervención y construcción comunitaria. Reforzar la atención primaria y los servicios sociales de base y dotarlos de recursos para la acción comunitaria puede ser un primer paso hacia la construcción de un sistema de bienestar que integre en mayor medida a la comunidad.

\subsubsection{Fomento del asociacionismo y el voluntariado}

Un cambio de valores es necesario si se pretende dotar de responsabilidades a las comunidades y a las personas que las integran. Si los propios individuos no sienten la necesidad y el valor de participar en la mejora de sus entornos y sus condiciones de vida, no será posible llevar a cabo políticas de empoderamiento comunitario que obtengan resultados razonablemente positivos.

La educación juega un papel fundamental, ya que las personas que no hayan sido inoculadas con el virus de la participación y no lo sientan como necesario desde jóvenes, es difícil que sientan la necesidad de hacerlo 
participar cuando sean adultos. Más hoy día, con el individualismo y la asociabilidad que caracterizan las sociedades occidentales, se hace necesario educar para la participación y la vida en comunidad.

Las asociaciones funcionan a menudo como escuelas de ciudadanía y de ciudadanos/as activos/as y participativos/as, por lo que el fomento y el desarrollo del tejido asociativo puede tener consecuencias positivas en el fortalecimiento de las comunidades, en tanto que ofrecen una red de oportunidades para participar y crecer en comunidad. De esta forma, tanto la educación en valores desde las escuelas como el desarrollo de las redes asociativas existentes en las comunidades pueden ser dos opciones complementarias para el desarrollo de personas activas y participativas que sean capaces y sientan la necesidad de asumir responsabilidades para la mejora de sus comunidades y condiciones de vida.

\subsubsection{El 'auzolan' y la gestión común de los bienes}

A la vista del escaso empoderamiento comunitario logrado en las experiencias analizadas y en la búsqueda de fórmulas alternativas que aumenten el papel de las comunidades en la provisión de bienestar, el debate que se plantea en torno a los bienes comunes puede servir no solo de sustento teórico, sino también de inspiración. Tal y como se ha expuesto a lo largo del artículo, abrir el debate en torno a formas de gestión más allá de lo público y lo privado, en la que las comunidades o grupos locales puedan tener un papel importante, se considera esencial a este respecto. Esta forma de gestión grupal, o de autogestión de un bien o servicio por parte un grupo o comunidad, puede ser una opción no solo para el mantenimiento o gestión de recursos o bienes naturales, sino también en ámbitos directamente relacionados con la protección social.

La práctica del auzolan, arraigada y desarrollada en el territorio vasco, es un claro ejemplo de gestión común, que si bien ha ido perdiendo fuerza a lo largo de los años, sigue vigente y en una fase de reverdecimiento. Siguiendo a Mitxeltorena (2011), en diferentes trabajos etnográficos sobre el País Vasco se constata la importancia que ha tenido el barrio (auzoa) para este pueblo. El barrio ha sido formado para dar respuesta a aquello a lo que la casa no ha podido dar respuesta por sí misma y, en la medida en que el barrio fue rellenando espacios y necesidades, se fue fortaleciendo (Mitxeltorena, 2011). Esta forma ancestral de organización y protección social que tan importante ha sido en el contexto vasco puede ser hoy día una clave determinante para el diseño de políticas de activación comunitaria.

La cultura del auzolan y de la autogestión sigue vigente en el contexto vasco y las experiencias que se desarrollan cada año en el País Vasco dan cuenta de ello. Tal y como se ha expuesto en capítulos anteriores, el asociacionismo y la vida en común están en declive y las presiones que ejercen los procesos descritos sobre los individuos son muy fuertes. Aprovechar la tradición y la inercia comunitaria que existe en el territorio puede ser una buena estrategia para buscar fórmulas alternativas y novedosas de dar respuesta a necesidades sociales. El auzolan sirve no solo de inspiración, sino también de eje sobre el que se puedan sostener diversas actividades o iniciativas relacionadas con la protección social.

Todas estas propuestas surgen en un contexto complejo para el desarrollo de dinámicas o estrategias de acción comunitaria. El individualismo y la carencia de tiempo y energía para asumir el trabajo comunitario después de las jornadas laborales, tareas domésticas o de cuidados, etc. hacen pensar que las comunidades no tienen actualmente la capacidad para asumir por ejemplo, la gestión de algún servicio que implique una dedicación considerable.

\section{Consideraciones finales}

Desde su surgimiento, el Estado de Bienestar ha sido testigo de múltiples transformaciones. La crisis financiera iniciada en el año 2008 fue el detonante de una profunda crisis en él, llegando a marcar un antes y un después en su proceso de desarrollo. Además, la existencia de nuevos riesgos sociales tales como la globalización, la mercantilización y la financiarización económica repercuten negativamente en un adecuado desarrollo del Estado de Bienestar, cuestionando así su utilidad y viabilidad.

Es bajo este contexto de incertidumbre en el que se plantea al Estado de Bienestar la pregunta compleja de cómo abordar la realidad de los cuidados y su gestión, introduciendo los elementos comunitarios que se han mencionado con anterioridad, y de cuáles tendrían que ser las estrategias para abordarlo. 


\section{Bibliografía referenciada}

ARARTEKO (2010): La situación de los Servicios Sociales de Base en la Comunidad Autónoma de País Vasco, Vitoria.

- (2016): La situación de los Servicios Sociales de Base en la Comunidad Autónoma de País Vasco, Vitoria.

BARBERO, J. (2010): "Retos metodológicos en Trabajo Social comunitario", en MARTINEZ, E. y PEÑA, L., Redefiniendo el Trabajo Comunitario, Vitoria, Escuela Universitaria de Trabajo Social, pp. 65-94.

BAUMAN, Z. (2003): Comunidad, Madrid, Siglo XXI de España Editores.

- (2011): Vida líquida, Madrid, Paidós.

BOLLIER, D. (2016): “El ascenso del paradigma de los bienes comunes", en HESS, C. y OSTROM, E., LoS bienes comunes del conocimiento, Quito, IAEN, pp. 51-64.

CASTRO-COMA, M.; y MARTÍ-COSTA, M. (2016): “Comunes urbanos: de la gestión colectiva al derecho a la ciudad”, EURE, 42, pp. 131-153.

CHANAN, G.; y MILLER, C. (2011): “The Big Society: How it Could Work?", en M. STOTT, The Big Society challenge, Norfolk, Keystone Development Trust Publications, pp. 52-64.

CIVIL EXCHANGE (2015): Whose Society? The final Big Society audit, DHA.

CORNWALL, A. (2002): “Locating Citizen Participation”, IDS Bulletin, 33, pp. 49-58.

DALY, M.; y LEWIS, J. (2000): "The Concept of Social Care and the Analysis of Contemporary Welfare States", The British Journal of Sociology, 51, pp. 281-298.

DEL PINO, E.; y RUBIO, M. (2016): Los Estados de Bienestar en la encrucijada. Políticas sociales en perspectiva comparada, Madrid, Tecnos.
DELSEN, L. (2016): The realisation of the participation society. Welfare state reform in the Netherlands: 2010-2015, Institute for Management Research, Radboud University.

ESPING- ANDERSEN, G. (1993): Los tres mundos del Estado de Bienestar, Valencia, Institució Alfons el Magnanim.

ESPING- ANDERSEN, G.; y PALIER, B. (2011): Los tres grandes retos del Estado de Bienestar, Barcelona, Ariel.

EVANS, K. (2011): "Big Society in the UK: A Policy Review", Children and Society, 25, pp. 164-171.

EVANS, M., MARSH, D.; y STOKER, G. (2013): “Understanding localism”, Policy Studies, 34, pp. 401-407.

FANTOVA, F. (2014): Diseño de políticas sociales, Madrid, CCS.

FERNÁNDEZ-GARCÍA, T. (2012): "El Estado de Bienestar frente a la crisis política, económica y social”, Portularia, 12, pp. 3-12.

FONDEVILA, G. (2003): "Política de derechos de "bien común'. Tres críticas posibles al comunitarismo, Diánoia, 50, pp. 85-109.

GÓMEZ, L. (2000): “Cooperativa y comunidad”, Arbor, 652, pp. 697-714.

GUTIÉRREZ- RUBÍ, A. (2012): “El procomún y la respuesta a la crisis”, Documentación Social, 165, pp. 103-117.

HESS, C., y OSTROM, E. (2016): “Introducción: una visión general de los bienes comunes del conocimiento", en HESS, C. y OSTROM, E., LoS bienes comunes del conocimiento, Quito, IAEN, pp. 27-50.

KISNERMAN, N. (1990): Comunidad. Teoría y Práctica del Trabajo Social, Buenos Aires, Humanitas.

KOSTER, M. (2014): “Bridging the gap in the Dutch participation society. New spaces for 
governance, brokers, and informal politics", Etnofoor, 26, pp. 49-64.

LOWNDES, V.; y PRATCHETT, L. (2012): “Local Governance under the Coalition Government: Austerity, Localism and the 'Big Society"', Local Government Studies, 38, pp. 21-40.

MAINO, F. (2015): “El segundo welfare entre innovación e inversión social: ¿cuáles son las respuestas a la crisis del Estado social?", en BRONZO, Z.; y REPPETO, F., "Coordinación de políticas sociales: desafíos para la gestión pública", Eurosocial, 18, Madrid, pp. 105-134.

MARCHIONI, M. (1999): Comunidad, participación y desarrollo: teoría y metodología de la intervención comunitaria, Madrid, Editorial Popular.

MATTEI, U. (2013): Bienes comunes, Bolonia, Trotta.

MESTRUM, F. (2016): Los comunes sociales. Ideas para la justicia social en el pos-neoliberalismo, Petaling Jaya, Gerakbudaya Digital Sdn Bhd.

MITXELTORENA, J. (2011): Auzolanaren kultura. Iraganaren ondarea, orainaren lanabesa, etorkizuneko giltza, Tafalla, Txalaparta.

MORENO, L. et al. (2014): Los sistemas de bienestar europeo tras la crisis económica, Madrid, Programa Eurosocial.

- (2012): La Europa asocial, Barcelona, Debate.

MOULAERT, F. (2010): "Social innovation and community development: concepts, theories and challenges", en MOULAERT, F. et al., Can neighbourhoods save the city? community development and social innovation, Abingdon, Routledge, pp. 4-16.

NAVAL, C.; y SISON, A. J. (2000): “Las propuestas comunitaristas en América y en Europa”, Arbor CLXV, 652, pp. 589-611.

NEWMAN, J.; y TONKENS, E. (2011): Participation, responsability, and choice: Summoning the active citizen in western european welfare states, Amsterdam, Amsterdam University Press.

NOGUÉS, L.; y GARCÍA, T. (2014): "Seducidos por lo común”, Trabajo Social Hoy, 73, pp. 43-62.
RAMOS, C. (2000): "Enfoque comunitario, modernidad y posmodernidad. El trabajo social con la comunidad en tiempos de la globalización", Cuadernos de Trabajo Social, 8, pp. 185-204.

RAZAVI, S. (2007): The political and social economy of care in a development context conceptual issues, research questions and policy options, serie Gender and Development Programme Papers, 3, Ginebra, United Nations Research Institute for Social Development.

REBOLLO, O.; y MORALES, E. (2013): "Hacia una política pública de acción comunitaria: límites y oportunidades", en CANAL, R., Ciudades y pueblos que puedan durar. Políticas locales para una nueva época, Barcelona, Icaria, pp. 303-316.

SCHINKEL, W. (2010): "The virtualization of citizenship", Critical Sociology, 36, pp. 265-283.

SENNETT, R. (2000): La corrosión del carácter, Barcelona, Editorial Anagrama.

SEOANE, C.; y RIAL, P. (2001): “Asociaciones ciudadanas y desarrollo comunitario", en MARCHIONI, M., Comunidad y cambio social, teoría y praxis de la acción comunitaria, Editorial Popular, pp. 165-18o.

SMITH, M. J. (2011): "The Intellectual Roots of Big Society", en STOT, M., The Big Society Challenge, Norfolk, Keystone Development Trust Publications, pp. 27-35.

TAYLOR-GOOBY, P. (2000): Risk, Trust and Welfare, Palgrave Macmillan.

VELÁSQUEZ, C. A. (2008): "El ir y venir de las ideologías políticas y el surgir del comunitarismo", Reflexión Política, 10, pp. 92-112.

VISCARRET, J. J. (2007): Modelos y métodos de intervencion en Trabajo Social, Madrid, Alianza Editorial.

WYLER, S. (2011): "Building the Big Society - Solid Foundations or Shifting Sands?", en STOTT, M., The Big Society Challenge, Norfolk, Keystone Development Trust Publications, pp. 36-42.

ZUBERO, I. (2012): “De los 'comunales' a los 'commons': la peripecia teórica de una práctica ancestral cargada de futuro", Documentación Social, 165, pp. $15-48$. 